Arteterapia. Papeles de arteterapia y educación para inclusión social ISSN-e 1988-8309

https://dx.doi.org/10.5209/arte.62284

\title{
Educación comunitaria a través de graffiti y arte urbano con jóvenes: investigación-acción y etnografía visual en Collblanc-La Torrassa (L'Hospitalet de Llobregat)
}

Pere Grané Feliu ${ }^{1}$

Recibido: 6 de noviembre 2018 / Aceptado: 18 de abril 2019

Resumen. Este artículo presenta los principales avances de una investigación sobre educación comunitaria a través del graffiti y el arte urbano con jóvenes, realizada entre 2014 y 2018 en el distrito Collblanc-La Torrassa de L'Hospitalet de Llobregat. La investigación, llevada a cabo en el marco de una tesis doctoral, empleó dispositivo metodológico compuesto por la investigación-acción y la etnografía visual. El artículo describe cómo se realizó la investigación y expone una selección de las evidencias recogidas más significativas. En particular, las contribuciones de este tipo de procesos educativos tienen que ver con el aumento de la identificación colectiva con el territorio, la promoción del empoderamiento juvenil y el fomento del trabajo en red en las comunidades.

Palabras clave: Educación comunitaria; investigación-acción; etnografía visual; jóvenes; arte urbano.

[en] Community education through graffiti and street art with youths: action research and visual ethnography in Collblanc-La Torrassa (L'Hospitalet de Llobregat)

\begin{abstract}
This article presents the main advances of a research on community education through graffiti and urban art with youths, developed between 2014 and 2018 in the Collblanc-La Torrassa district of L'Hospitalet de Llobregat. The research, carried out within the framework of a doctoral thesis, used a methodological device composed of action research and visual ethnography. The article describes how the research was conducted and exposes a selection of the most significant evidence collected. In particular, the contributions of this type of educational processes are related to the increase of collective identification with the territory, the promotion of youth empowerment and the strengthening of networking in communities.
\end{abstract}

Keywords: Community education; visual ethnography; youth people; graffiti; street art.

Sumario. 1. Introducción; 2. Jóvenes, arte urbano y educación comunitaria; 3. Investigación-acción y etnografía visual como métodos emancipadores y participativos; 4. Selección de evidencias del proceso que dio lugar a un mural contra el racismo; 5 . Contribuciones principales de la educación comunitaria a través del graffiti y el arte urbano; 6. Referencias bibliográficas.

Cómo citar: Grané Feliu, P. Educación comunitaria a través de graffiti y arte urbano con jóvenes: investigación-acción y etnografía visual en Collblanc-La Torrassa (L'Hospitalet de Llobregat), en Arteterapia. Papeles de arteterapia y educación para inclusión social 14, 2019, 3-19.

1 Doctor en Ciencias de la Educación de la UAB y miembro del grupo Equipo de Investigación sobre Diversidad e Inclusión en Sociedades Complejas (ERDISC, en catalán).

E-mail: peregranefeliu@gmail.com 


\section{Introducción}

En este artículo se pretende responder a la pregunta siguiente: ¿cuáles son las contribuciones principales de los procesos de educación comunitaria mediante graffiti y arte urbano con jóvenes? En coherencia con esto, la hipótesis general sostiene que el desarrollo de procesos basados en la educación comunitaria a través del graffiti y el arte urbano con jóvenes permite conseguir un conjunto de contribuciones positivas. Mediante una investigación realizada entre 2014 y 2018, se analizan procesos participativos que se impulsaron con agentes de Collblanc-La Torrassa (L'Hospitalet de Llobregat) relacionados con la creación de una intervención visual de tipo mural en un espacio urbano del distrito. El dispositivo metodológico empleado estuvo compuesto por la combinación de dos métodos: la investigación-acción y la etnografía visual. Anteriormente, se publicó un artículo donde se abordaba el proceso inicial de esta investigación (Grané, Rifà-Valls y Essomba, 2017). En esta ocasión, se pretende sintetizar los principales avances de la tesis doctoral: Jóvenes, Arte Urbano y Educación Comunitaria: Investigación-Acción y Etnografía Visual en el Distrito Collblanc-La Torrassa (L'Hospitalet de Llobregat) ${ }^{2}$. En cuanto a la estructura del artículo, primeramente, se expondrán las definiciones de los ámbitos que orientaron la investigación; a continuación, se incluirá una aproximación al dispositivo metodológico; posteriormente, se presentará una selección de las evidencias recogidas más significativas; y finalmente, se expondrán las principales contribuciones.

\section{Jóvenes, arte urbano y educación comunitaria}

Los conceptos principales empleados en esta investigación están vinculados con la pregunta presentada anteriormente. En particular, el ámbito de la investigación es la educación comunitaria; el objeto de estudio es el graffiti y el arte urbano; y los sujetos participantes son las personas jóvenes. A continuación, se expondrán aproximaciones teóricas para cada uno de estos conceptos.

El concepto de «educación comunitaria» (a partir de ahora EC) conlleva incorporar la perspectiva comunitaria en los procesos educativos. Es decir, considerar: la inscripción territorial próxima, las relaciones y lazos afectivos comunes y pautas específicas de interacción social. Estos elementos contextuales influyen en la cohesión y también en el sentimiento de identificación de las personas que viven en un territorio. En coherencia con esto, la «comunidad» se entiende "como una agrupación de personas e instituciones que se inscriben en un territorio y entre las que hay una serie de relaciones e interés en común" (Civís y Riera, 2007: 41). Concretamente, esta concepción de la comunidad enfatiza las relaciones y lazos afectivos que existen en un entorno de proximidad. La existencia de un espacio común, con lazos afectivos entre personas y relaciones entre agentes, no es un a priori, sino un proceso de construcción que se caracteriza por la interacción (Mayugo, Pérez y Ricart, 2004). La EC es fruto de la acción educativa en red que se da desde la comunidad, para la comunidad y con la comunidad como principal protagonista (Civís, 2005).

2 La tesis fue dirigida por Montserrat Rifà-Valls y Miquel Àngel Essomba Gelabert y contó con el apoyo de la Secretaria d'Universitats i Recerca del Departament d'Economia i Coneixement de la Generalitat de Catalunya y el Fondo Social Europeo. 
Calificar acciones y procesos educativos como EC implica comprometerse con el logro de tres elementos: la concientización, el empoderamiento y el trabajo en red. Según Freire (1970), «la concientización» hace referencia a procesos de conciencia crítica de reflexión y acción sobre la realidad para transformarla. Por otro lado, el «empoderamiento» está relacionado con la organización comunitaria y el fortalecimiento político, tanto individual como colectivo (Úcar, Jiménez-Morales, Soler y Trilla, 2016). No se trata de empoderar las personas, sino que sean ellas las que, a través de procesos de concientización se empoderen. Finalmente, el «trabajo en red» consiste en promover relaciones estables basadas en la horizontalidad, la corresponsabilidad y el interés mutuo (Civís y Longás, 2015). Así, en la EC la participación de cada persona y agente es necesaria y, al mismo tiempo, necesitada por los demás para cuestionar las relaciones de poder tradicionales (Tort y Collet, 2017). Finalmente, para que la EC sea realmente inclusiva debe buscar adoptar un enfoque que integre las desigualdades múltiples: de género, origen, clase, edad, orientación sexual, capacidad funcional, religión, entre otras.

El segundo concepto vertebrador del marco teórico es el de «jóvenes». Tradicionalmente, se ha asociado la «juventud» con un período vital transitorio ubicado entre la dependencia infantil y la autonomía adulta (Feixa, 1998). Actualmente, entender este segundo concepto como una «etapa vital provisional» resulta más complicado porque la juventud se ha postergado y se han borrado las barreras que la definían debido a factores de tipo económico, político, social y cultural. Uno de los elementos que tiene en común la juventud es la pérdida de autonomía y el mantenimiento de la dependencia de personas que deben seguir un camino establecido para acceder a la condición de persona adulta y «plena ciudadanía». Según Comas (2011), durante tres décadas en toda Europa las personas jóvenes se sacrificaban a la espera de convertirse en personas adultas activas -emancipadas, con autonomía y, en el caso español, piso de propiedad-. Se trataba de una emancipación idealizada a costa de no asumir alternativas, proyectos sociales o políticos propios. Muchos de los factores que hoy en día dificultan la emancipación de la juventud están asociados a la precariedad. La precariedad comporta un aumento de la temporalidad laboral, exige una formación continuada al largo de la vida y disminuye las garantías materiales que permiten tomar las propias decisiones. En particular, uno de los elementos clave de la juventud es el tiempo libre para el ocio porque representa un canal de expresión de las identidades de las personas jóvenes. Mientras que disponer de menos responsabilidades en el trabajo tiene que ver con factores de clase, en todo lo relativo a las responsabilidades familiares tiene un mayor peso los factores de género. Según Moreno (2015), la persistencia de las desigualdades de género en la distribución del trabajo doméstico pone límites a la emancipación y al cambio generacional.

Además de estas dificultades en el mercado laboral, también se deben tomar en consideración elementos de tipo cultural, como por ejemplo la dependencia familiar. En el Estado español, la evidencia de que en períodos de crecimiento económico la edad de emancipación juvenil fuera igualmente elevada tiene que ver con el modelo familiar que comparten las sociedades europeas de la mediterránea. Se trata del «régimen de bienestar familista», que se caracteriza por debilidades estructurales en el mercado laboral y que tiene como consecuencia que las políticas sociales se dejan en manos de las familias (Serracant, 2013). En estas sociedades las familias asumen la protección juvenil con el argumento que las personas jóvenes son proyectos de personas adultas (Comas, 2000). A cambio de que no se cuestione la autoridad de las 
familias, estas asumen el papel en el acompañamiento y orientación para dirigirlas a la condición adulta. La juventud se sitúa en una relación de dependencia familiar de acuerdo con una jerarquía adultocéntrica, donde las posiciones de poder de dominio se concentran en los adultos (Duarte, 2012). La «juventud» es un agregado heterogéneo de agrupaciones de individuos en los que la precariedad se distribuye de forma desigual, pero que comparten la identidad social de espera pasiva para transitar de una situación a otra (Comas, 2015).

Los últimos conceptos que fundamentan la investigación son graffiti y arte urbano. El término «graffiti» hace referencia al movimiento cultural originado a finales de la década de los años 60 en la ciudad de Filadelfia cuando escritores/as ${ }^{3}$ históricos como Cornbread y Cool Earl popularizaron la tradición de realizar firmas. Este fenómeno cobró un gran impulso cuando llegó a la ciudad de Nueva York, especialmente en el momento en que las firmas de graffiti tomaron el control visual de los trenes de metro durante la década de los 70 (Wacławek, 2011). La aparición del graffiti, también conocido como «graffiti neoyorkino» (Abarca, 2010), estuvo fuertemente relacionada con el abandono urbano de determinados distritos (Austin, 2001; Chang, 2007). La crisis urbana de los años 60 dio lugar a un éxodo de las clases medias al extrarradio de ciudades como Filadelfia o Nueva York que, en la década posterior, dejó muchos lugares abandonados y espacios libres donde firmar (Figueroa, 2014). Así, el graffiti, junto con otros movimientos culturales contemporáneos, mantiene una fuerte relación con el contexto urbano más cercano porque es donde se materializan las desigualdades múltiples. En Nueva York, durante la década de los 80 se promovieron los escritores/as de graffiti como nueva tendencia artística, a pesar de que las personas que expusieron de forma continuada en galerías fueron pocas, por ejemplo: Crash, Daze o Lady Pink. Los medios de comunicación eclipsaron estas contribuciones con obras de Jean-Michel Basquiat o Keith Haring, quienes habían hecho una transición del graffiti no autorizado al arte convencional. En algunas ciudades estadounidenses esto se sumó a la tradición importada del fresco político del muralismo chicano, fuertemente influenciado por el movimiento de los derechos civiles (Latorre, 2008). Durante las décadas de los 80 y los 90 el graffiti se desarrolló exponencialmente y se expandió mundialmente a través de los medios de comunicación y de películas como Wild Style, Beat Street o Style Wars.

En el Estado español las primeras muestras de graffiti aparecen a principios de la década de los años 80. Según Berti (2010), las ciudades donde el graffiti cobra más fuerza son: Alicante, Barcelona y Madrid. En Madrid, surgieron dos corrientes diferenciadas que convergieron a finales de esta década: el graffiti autóctono madrileño o graffiti flechero, liderado por Muelle, y el graffiti de tradición neoyorquina. No fue hasta 1985 que la presencia de graffiti acabó siendo habitual en las ciudades citadas. En el área metropolitana de Barcelona los primeros grupos aparecen a mediados de los años 80. La posterior efervescencia de los años 90 situó a Barcelona entre las ciudades más destacadas de Europa, motivo por el cual artistas de renombre internacional, como por ejemplo Banksy, visitaron la ciudad. La situación se frenó drásticamente en 2005 con la aprobación de las llamadas "Ordenances de Civisme". Esta legislación incluye un capítulo específico, llamado "Degradació Visual de l'Entorn Urbà", que penaliza el graffiti no autorizado y establece sanciones que pueden llegar

Persona que practica graffiti. Originariamente se hacían llamar escritores porque escribían su nombre por doquier. 
a los $3.000 €$ para los infractores. Esto se enmarca en aquello que se conoce como «wars on graffiti» (Dickinson, 2008), un conjunto de medidas que han comportado un aumento del gasto y el uso de métodos más sofisticados para erradicar de forma violenta todo aquello que induce al «desorden» visual. El graffiti, implícitamente, establece un desafío a las relaciones urbanas y constituye un acto transgresor contra la propiedad (McAuliffe, 2012). A pesar de que el graffiti pueda no corresponderse con mensajes visual y políticamente inteligibles, no carece de motivación política. Según Menor (2015), se trata de un reclamo del espacio público, ya que se basa en hacer un uso radicalmente diferente de la ciudad.

Desde que se popularizó el graffiti neoyorquino se han producido diversos cambios que han dado lugar a movimientos culturales diferentes, de los cuales destaca el arte urbano o «street art». El arte urbano se trata de un movimiento que se dirige al público general, que emplea técnicas diversas (plantillas, carteles, pegatinas, etc.) y que surge del encuentro entre el arte contemporáneo con diversas formas de cultura popular, en particular el graffiti (Abarca, 2012). Por último, tanto en el arte urbano como en el graffiti existen sesgos de género que inciden negativamente en la participación del género femenino porque en ambos movimientos se estigmatizan, cosifican y se adoptan actitudes paternalistas hacia las mujeres (Macdonald, 2001; Lombard, 2014; Recinos, 2015). Todavía hoy, las mujeres se ven obligadas a esforzarse mucho más que los hombres para gozar de la misma aceptación (Sany, 2016). En consecuencia, estos movimientos culturales están impregnados del modelo de masculinidades hegemónico, las estructuras propias del patriarcado y su configuración del espacio público (López, 2015; Rodó-de-Zárate, 2016)

\section{Investigación-acción y etnografía visual como métodos emancipadores y participativos}

El dispositivo metodológico utilizado en la investigación se compone de dos métodos: la investigación-acción y la etnografía visual. Ambos métodos comparten un conjunto de atributos que fortalecen el dispositivo metodológico y le dan coherencia. En primer lugar, consideran la participación como una estrategia para la investigación y el empoderamiento. Se trata de hacer investigación con las personas participantes en lugar de hacerla sobre ellas (Clark, 2010; Pink, 2011). Segundo, ambos permiten el conocimiento democrático. No consiste en utilizar una receta técnica para profundizar en la democracia, sino incorporar principios democráticos en la investigación (McNiff, 1988). En tercer lugar, contribuyen a la ciencia social y al cambio social. La investigación persigue el cambio educativo e interrelaciona consciente y dialécticamente la teoría y la práctica.

La investigación acción (a partir de ahora I-A) consiste en promover espirales de autorreflexión que, tradicionalmente, pasan de forma cíclica por las siguientes fases: planificación, acción, observación y reflexión (Lewin, 1946). La I-A debe promover un conjunto sistemático de acciones, pero también de aprendizajes. No se trata de exponer las acciones realizadas, sino el conocimiento asociado a ellas. Según McNiff (1988), el proceso de desarrollo de conocimiento debe ser espontáneo, sin que

4 Para más información sobre los sesgos de género en el graffiti y el arte urbano consulta: Grané, Rifá-Valls y Essomba, 2017; o Grané, 2018. 
las fases deban darse siguiendo un orden secuencial. Esto permite que el facilitador de la investigación afronte diversos problemas al mismo tiempo sin perder de vista la cuestión central, cosa que hace posible abordar el problema principal desde nuevas dimensiones. Estos planteamientos metodológicos se concretaron en procesos de diálogo en los que participaron distintos agentes de la comunidad (grupos de jóvenes, agentes técnicos, artistas, etc.) siguiendo la noción de «pacto sociocultural» de Úcar (2009). La planificación fue acordada colectivamente y en ella se constataron cuáles eran los intereses y demandas de cada agente y cuál sería su colaboración en el proceso. Esto fue fruto de ciclos participativos impulsados por agentes técnicos de la comunidad. Los principales motivos por los cuales los agentes técnicos acabaron asumiendo el rol de impulsores tienen que ver con su conocimiento, infraestructura, disponibilidad horaria y vínculos previos con la comunidad. De acuerdo con lo anterior, estos ciclos se desarrollaron siguiendo el esquema siguiente:

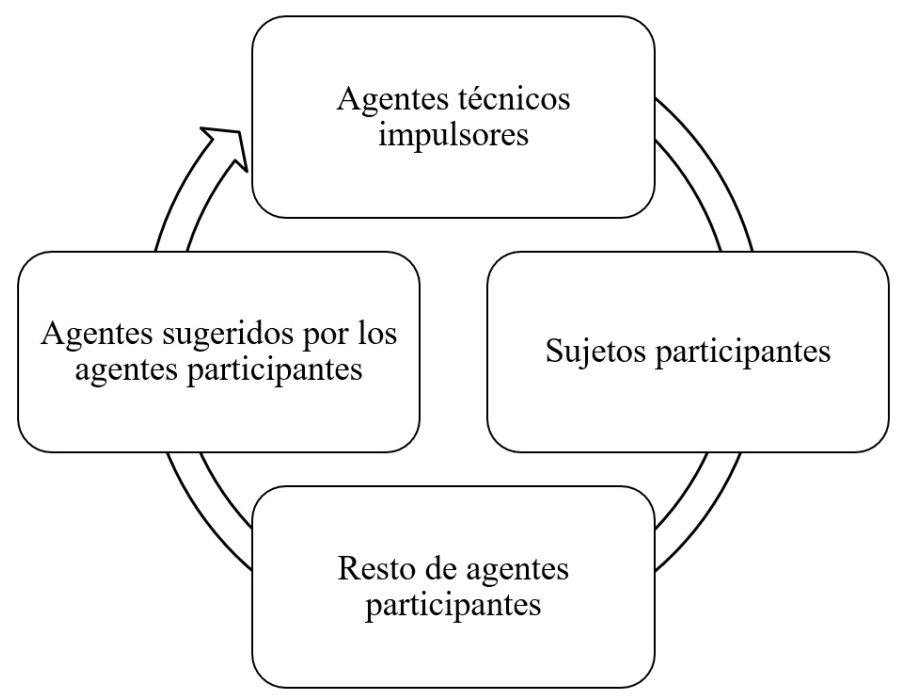

Figura 1. Construcción colectiva de la propuesta de acción. Elaboración propia.

La tarea de la facilitación consistió en hacer de bisagra entre agentes de la comunidad para incluir el mayor número de agentes y evitar que ninguno dejara de ser parte de los procesos por no disponer de información suficiente. Los aspectos considerados más positivos de la tarea de facilitación por parte de los agentes participantes fueron: presentar de forma clara el planteamiento de la investigación a todos los agentes; vehicular la investigación a través de espacios de coordinación previamente existentes; favorecer que cada agente pudiera participar de formas diferentes; prever tiempo suficiente para desarrollar cada una de las fases de la planificación acordada; respetar los ritmos de los agentes involucrados; fomentar la suma de líneas de acción educativa para evitar su solapamiento; y hacer de contacto entre agentes de la comunidad.

El segundo método empleado fue la etnografía visual (de ahora en adelante EV). En la EV el investigador/a se involucra en la observación, al mismo tiempo que participa en el establecimiento de relaciones con aquello que investiga y se focaliza en cómo 
la experiencia y la práctica forman parte de procesos globales. Además, no se limita a utilizar métodos visuales en la investigación y la representación, sino que profundiza en la relación entre lo visual y otros elementos de la cultura, la sociedad y la experiencia (Pink, 2006). La EV permite ir más allá de la noción de «experto» cuando explora formas de conocimiento que a menudo están ocultas. Favorece hacer sentir aquellas experiencias, voces y sentimientos que pueden ser difíciles de verbalizar. El énfasis en la colaboración en el uso de este método ofrece la posibilidad de experimentar con diferentes tipos de construcciones narrativas para llegar a un público más diverso, cuestionar la autoridad narrativa e introducir análisis de la realidad social heterogéneos y complejos. De acuerdo con esto, en esta investigación se combinó el diario de campo, con entrevistas, grupos de discusión y evidencias visuales de diversos tipos: I) fotografías de rutas realizadas en la ciudad por parte del facilitador; II) documentación visual realizada por los educadores/as durante las acciones educativas; III) cartografías visuales de espacios urbanos significativos realizadas por los grupos de jóvenes; IV) diario audiovisual de los procesos de EC realizado por los grupos de jóvenes; V) reflexión y reconstrucción visual de procesos de EC por parte de un agente técnico; VI) fotografías para difundir los procesos de EC en las redes sociales; y VII) fotografías del facilitador para la reflexión sobre el uso de la etnografía. Todos estos niveles de la EV muestran la flexibilidad de este método a la hora de favorecer la participación en la recolección de las evidencias, al mismo tiempo que permite conectar con la visualidad del día a día de las personas participantes.

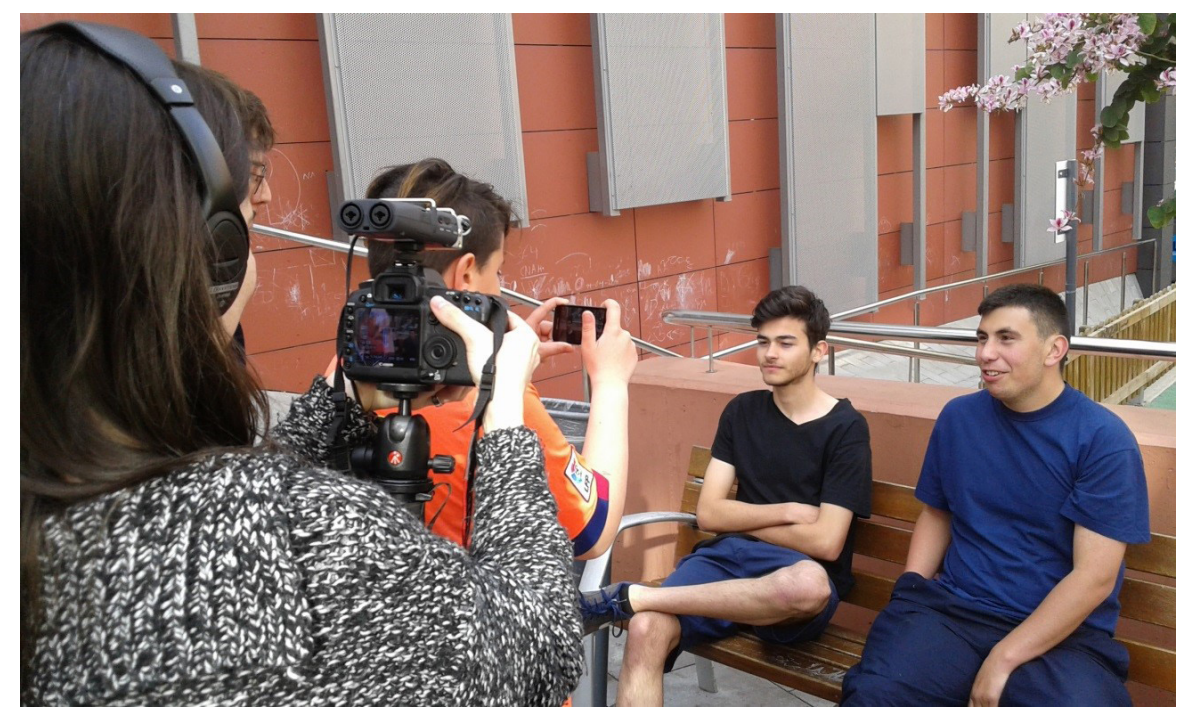

Figura 2. Distribución del rol etnográfico en el uso de técnicas visuales. Fotografía de Pere Grané.

Uno de los productos que mejor ejemplifica las potencialidades del uso de la EV es la memoria audiovisual del proceso de creación colectiva que hicieron los grupos de jóvenes en el marco de la investigación ${ }^{5}$. La elaboración de este documento audiovisual fue uno de los elementos clave de la investigación porque mostró la

Puedes consultar la memoria audiovisual en el enlace siguiente: https://vimeo.com/184362305 
coincidencia de diversas narrativas y voces sobre los procesos asociados a la investigación, más allá de la pintura del mural final. La edición de la memoria audiovisual supuso un reto a la hora de triangular la información y fue realizada por el facilitador con el apoyo de un agente que tenía un conocimiento elevado en el ámbito de la comunicación audiovisual. Este documento requirió tomar en consideración todas las evidencias visuales recogidas durante la investigación y hacer una síntesis coherente a través de una reflexión crítica. Además, permitió hacer un retorno de la investigación a públicos más amplios a través de un documento que invita a conocer de forma más amplia los procesos relacionados con la investigación. En la fase final de la investigación la memoria audiovisual fue presentada a la comunidad por parte de los grupos jóvenes participantes.

\section{Selección de evidencias del proceso que dio lugar a un mural contra el racismo}

En este apartado se exponen algunas de las evidencias más significativas de la investigación. El gran volumen de evidencias recogidas en el marco de la tesis doctoral no hace posible hacer una exposición exhaustiva. Teniendo en cuenta esto, a continuación, se hace una selección de los elementos más representativos del desarrollo de la investigación.

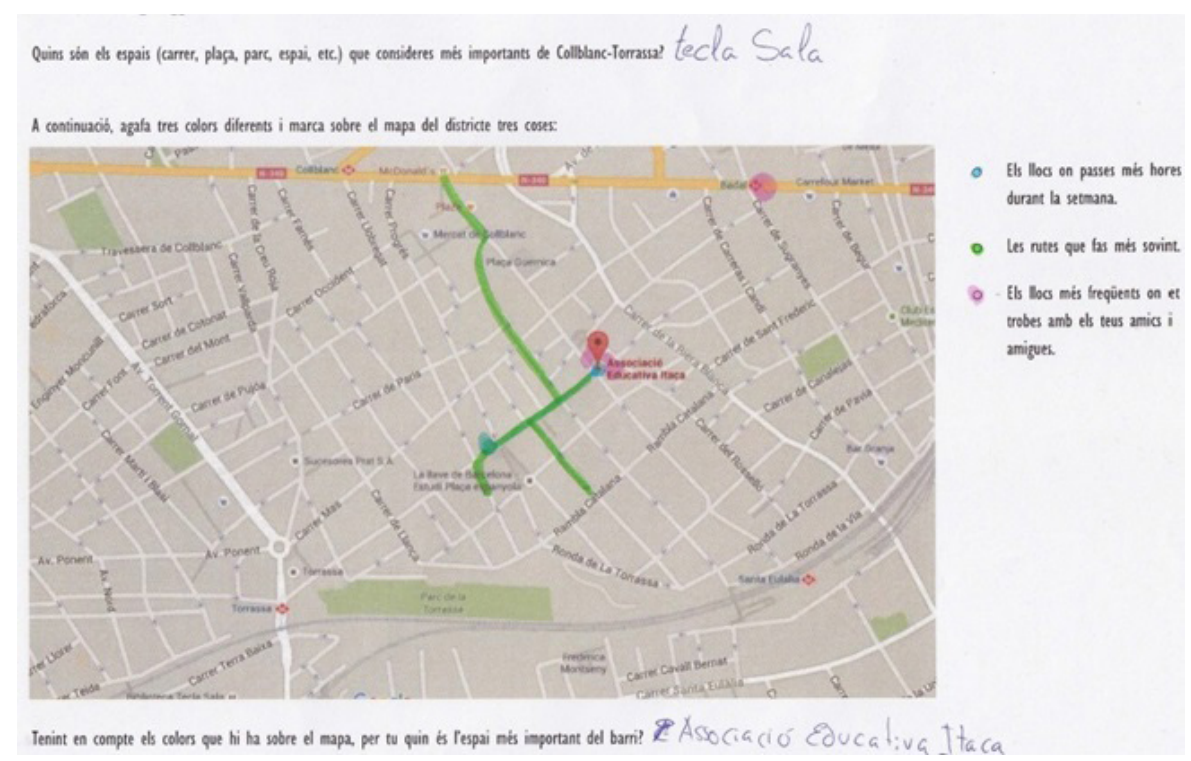

Figura 3. ¿Dónde pasas más horas, qué rutas haces a menudo y dónde te encuentras con tus amigos y amigas? Ficha realizada por un joven del espai jove.

En un primer estadio se realizó una detección de las necesidades con los grupos de jóvenes. En particular, participaron dos grupos que forman parte de una entidad educativa del distrito. Un grupo, el casal de joves, se correspondía con un servicio centrado en el refuerzo educativo después del horario de instituto. El otro, el espai jove, tiene por objetivo la orientación y el fomento del ocio durante el fin de semana. A través de acciones educativas de reflexión sobre los espacios urbanos del distrito 
en cuestión, se pretendía incluir las experiencias y condiciones de vida de las personas jóvenes. Al comienzo, cada grupo de jóvenes identificó los espacios que consideraba más significativos del distrito. Se usaron elementos de investigación que se basan en las cartografías sociales a la hora de aproximarse a estos espacios físicos y sociales (Juanola y Fábregas, 2011). Se facilitó un mapa de googlemaps del distrito para cada joven para que identificara los lugares donde pasa más horas durante la semana, las rutas que hace más a menudo y los espacios donde se encuentra con el grupo de iguales (ver figura 3). Esta información contribuyó a la detección de necesidades y orientó la posterior decisión relativa a la decisión del espacio donde realizar la intervención visual final.

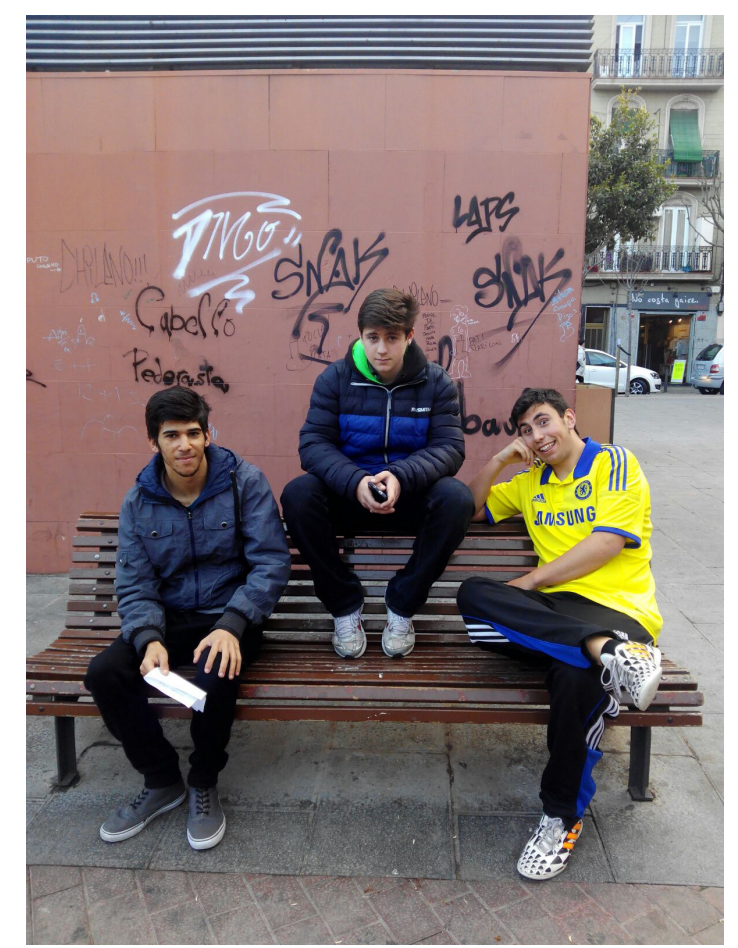

Figura 4. Apropiación funcional y visual (2016). Fotografía de Júlia Massot.

Sobre esta base, ambos grupos realizaron la etnografía visual participativa de los espacios identificados. Estas acciones fueron unas de las más trascendentes de la investigación. Mediante la fotografía, el instrumento visual más utilizado por la juventud para compartir sus vivencias (Marcellán-Barace, Calvelhe, Agirre y Arriaga, 2013), se estableció una relación horizontal y de diálogo entre iguales, en la que estas personas pudieron narrar lo que sucedía en sus vidas con un instrumento que dominaban. Los grupos de jóvenes se fotografiaron a sí mismos en los espacios urbanos identificados, cosa que permitió aflorar voces y sentimientos invisibles difíciles de verbalizar, pero que forman parte de la rutina de las personas participantes. Un ejemplo de esto se encuentra en la fotografía que realizaron en un banco de la plaza donde se realizó la intervención visual 
final (ver figura 4). Mediante esta fotografía, y la reflexión posterior que hicieron ambos grupos, se constató que las personas jóvenes mayormente utilizan el banco de la plaza que queda más oculto cuando hay menos gente, a última hora de la tarde. El uso intensivo de este rincón particular es una expresión de la vinculación de estas personas con el barrio donde viven (Zegrí, 2014). Tanto la plaza como el banco en cuestión se utilizan de formas diversas según la franja horaria. Tal y como sostiene Aramburu (2009), se producen especializaciones funcionales, pequeñas segregaciones en una red espacial y temporal, para dar respuesta a los diferentes tipos de personas usuarias.

Según Gutiérrez y Ciocoletto (2011), la planificación urbana atribuye un reparto desigual de las oportunidades para cubrir las diferentes necesidades, motivo por el cual hay colectivos que se ven obligados a apropiarse de los espacios urbanos para hacer un uso que responda a sus necesidades. En diversas fotografías realizadas por las personas jóvenes un elemento común fue la búsqueda de espacios de relación alejados del control de los adultos. Estas evidencias muestran de qué manera las personas jóvenes resignifican el espacio de manera que sean ellas las que definen las propias normas del espacio. En términos visuales, resulta relevante constatar que en los espacios apropiados por las personas jóvenes existe también una apropiación visual en forma de inscripciones, a través de la cual se expresan identidades y se da forma a la ciudad (Keith, 2005).

En acciones educativas posteriores se dinamizaron grupos de discusión con ambos grupos en los que se utilizaron las fotografías realizadas durante la etnografía visual participativa. Estas evidencias sirvieron para reflexionar sobre sus vivencias en estos espacios urbanos y, al mismo tiempo, se utilizaron como base para escoger el lugar y el tema de la intervención visual final. Durante las discusiones grupales las personas jóvenes manifestaron experiencias de racismo de forma recurrente:

Alguna vez oí algún comentario de familia sobre que «son negros», «no se duchan», «son más guarros» o que «por el carácter destacan más». Alguna vez me molesto porque son parte de mi familia; aunque yo sea mitad dominicana y mitad española me pongo en las dos situaciones. Tengo un amigo que le molesta que le digan «negro». Pero, por ejemplo, mi prima, que es negra, está orgullosa de serlo (Jaz, joven del espai jove).

La gente de otro país, como yo, se siente muy ofendida con la gente que está en este país porque nos insultan. Por ejemplo, a mí mucha gente me llama moro de mierda y eso a mí me enfada (Tariq, joven del casal de joves).

En un segundo período, se acordó con los grupos de jóvenes que el tema de la intervención visual estuviera relacionado con el racismo que vivían diariamente. En consecuencia, se dinamizaron acciones educativas diversas para iniciar un proceso reflexivo que, más adelante, se combinó con el proceso creativo de la intervención. La creación de la intervención visual final fue realizada por una artista callejera local y por un miembro de una entidad cultural local. Durante el proceso se reflexionó sobre los sentimientos que las personas participantes asociaban al racismo y sobre el mensaje que querían transmitir a través de la forma y el color (ver figura 5). 


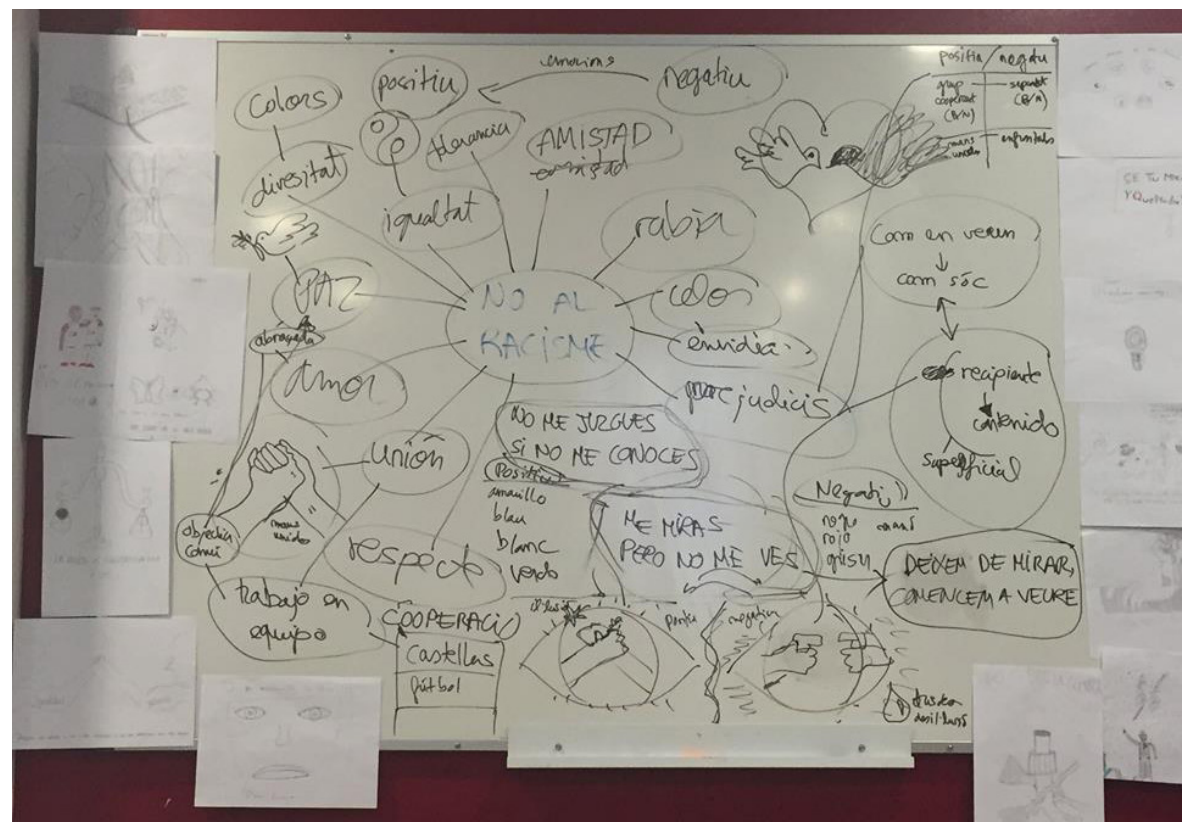

Figura 5. Proceso creativo de la intervención visual (2016).

Fotografía de Angie Carrasco.

Mediante acciones de expresión visual y plástica, más allá del dominio del espray de pintura, se conectó la curiosidad inicial que habían mostrado por el graffiti con un repertorio más amplio de lenguajes visuales y plásticos. La búsqueda de acuerdos conjuntos que permitieran proseguir en el proceso creativo no fue una tarea senci1la. Tal y como sostiene Sánchez de Serdio, "tratándose de proyectos basados en la participación y en la creación conjunta, ninguna propuesta de arte colaborativo puede presentarse como una secuencia aproblemática de incorporación de voluntades convergentes" (2008: 17). En el caso concreto de este proceso de creación colectiva hubo algunas limitaciones porque las personas adultas que asumimos la dinamización, ocasionalmente, tuvimos una incidencia excesiva en los procesos de toma de decisiones. Se priorizó alcanzar acuerdos de mínimos, en los que se agrupaban de opiniones parecidas, en lugar de debatir en profundidad sobre las diferentes vivencias del racismo. Teniendo en cuenta esto, el mensaje escogido por los grupos de jóvenes fue el siguiente:

También le queremos dar un mensaje a todos los chicos del barrio, jóvenes y mayores, de que, como pueden ver, no somos un grupo de la misma nacionalidad, sino que estamos mezclados. Algunos somos latinos, otros somos de aquí. Queremos darle el mensaje conforme no juzguéis un libro por su portada, sino llegar a conocer a la gente de verdad como es. No todos somos iguales. (Alan, joven del espai jove).

En el último estadio se realizó la pintura del mural y la evaluación del proceso. En la pintura participaron los dos grupos de jóvenes mencionados anterior- 
mente y, además, se sumaron otros jóvenes de la comunidad que habían visto acciones educativas previas que fueron realizadas en espacios urbanos abiertos. Durante la pintura, los jóvenes participantes supieron qué hacer porque habían participado en la planificación; las técnicas visuales no les eran desconocidas, ya que habían realizado acciones relacionadas con anterioridad; no mantuvieron una actitud pasiva ante el muro, sino que participaron activamente; y protagonizaron aquello que sucedió en el espacio urbano en cuestión. A diferencia de acciones educativas de pintura previas, las jóvenes de los grupos no se quedaron al margen, sino que participaron del mismo modo que los jóvenes (ver figura 5). La mayor participación del género femenino se dio cuando se implementaron las medidas siguientes: el fomento de acciones basadas en dinámicas colaborativas, el uso de técnicas y lenguajes visuales y plásticos diversos y la incorporación de referentes femeninos de graffiti y arte urbano en la dinamización de las acciones educativas.

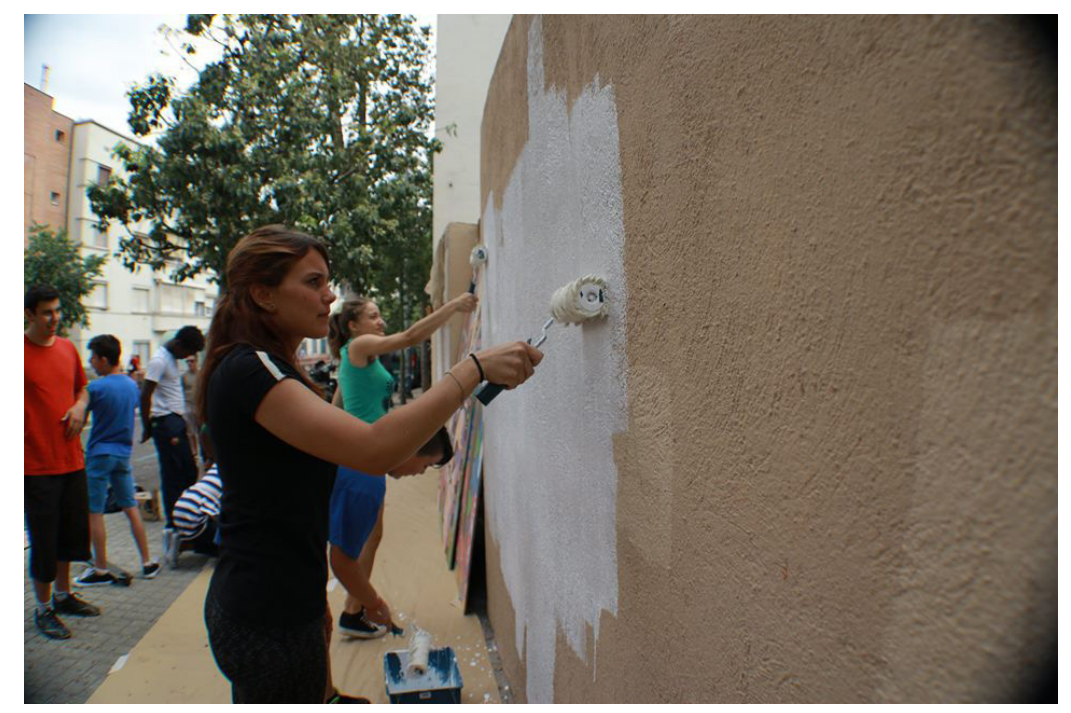

Figura 6. Empoderamiento femenino callejero (2016). Fotografía de Júlia Massot.

En los grupos de discusión que se realizaron en la evaluación final, las acciones educativas valoradas más positivamente por parte de los jóvenes fueron las que estuvieron relacionadas con el dibujo y la pintura, incluso por parte de personas que manifestaron que no les gustaba dibujar. En particular, el aspecto más destacado no fue el uso de técnicas visuales y plásticas, sino el trabajo colaborativo entre grupos jóvenes. "Lo que me gustó más de esta actividad fue, sobre todo, cuando hicimos el mural, ya que interactuamos entre todos. Compartimos más momentos con los del espai jove y los del casal de joves, que tampoco estamos mucho con ellos (Judith -joven del espai jove-). Así, durante el proceso el fomento de los espacios de encuentro basados en la informalidad, la autoexpresión y la autoorganización obtuvo buenos resultados. Por ejemplo, permitió sumar jóvenes que previamente no participaban en entidades educativas de la comunidad. En coherencia con Zegrí (2014), estas acciones fueron percibidas como más beneficiosas por parte de las 
personas jóvenes porque adquirieron más autonomía. La mayor participación juvenil se dio en las acciones educativas cercanas a los "procesos abiertos y creativos, orientados a crear espacios de trabajo no estructurados y que puedan contemplar los contenidos culturales con los que se trabaja, no como objetos que deben ser asimilados, sino como movimientos de apertura y reconfiguración de esos mismos procesos" (Marí, 2014: 31). Así lo confirmaron parte de los agentes técnicos participantes: "les gustaba cuando se hacían actividades no dirigidas [...]. En el caso del graffiti ha sido bastante fácil porque las actividades se han ido adaptando y cambiando de día" (educadora del casal de joves). Un ejemplo concreto fue el conjunto de exhibiciones de graffiti que se realizaron en el marco del proceso en espacios urbanos previamente identificados como significativos por los grupos de jóvenes participantes (ver figura 7).

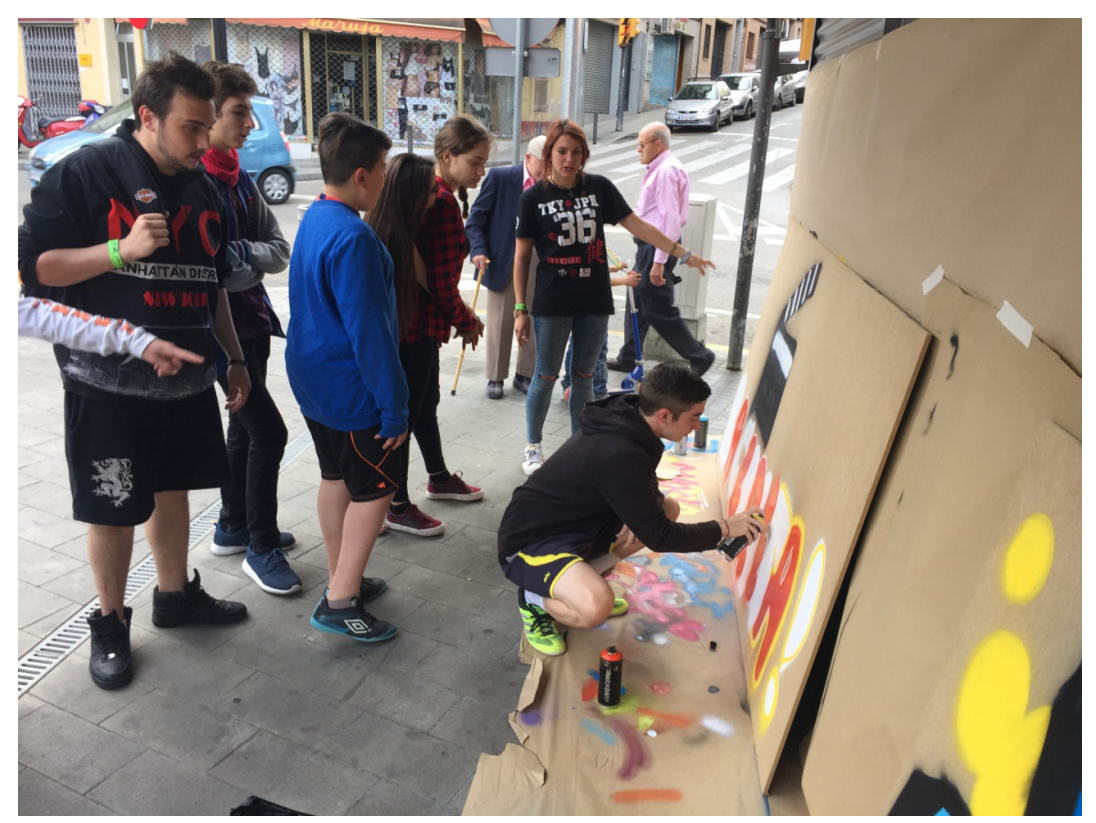

Figura 7. Espacios de encuentro y relación con la comunidad (2016). Fotografía de Angie Carrasco.

Finalmente, los procesos comunitarios asociados a la investigación no finalizaron con la evaluación. Durante el curso académico posterior el número de acciones educativas relacionadas con el graffiti aumentaron en el distrito y el proceso comunitario se diversificó en varios procesos. Los agentes técnicos acabaron desarrollando diferentes tipos de procesos: de continuidad, que reproducían acciones realizadas previamente; de complementariedad, que completaban carencias del proceso expuesto; y de transformación, que perseguían finalidades diferentes o se utilizaban en otros contextos. Un elemento clave que explica la sostenibilidad de los procesos fue el fomento de la inclusión de un gran número de agentes durante la investigación. Incluso aquellos agentes que habían tenido un papel poco activo, más adelante asumieron el rol de impulsores en nuevas acciones. 


\section{Contribuciones principales de la educación comunitaria a través del graffiti y el arte urbano}

Las evidencias expuestas en el apartado anterior muestran de qué manera el graffiti y el arte urbano pueden contribuir a fomentar la participación en procesos de EC. Además, este tipo de procesos permitieron mejorar: la identificación colectiva con el territorio, el empoderamiento juvenil y el trabajo en red en la comunidad.

En primer lugar, la EC a través del graffiti y el arte urbano favoreció que aumentara la identificación colectiva de las personas jóvenes participantes con el territorio. A través de la intervención visual, se generaron nuevas experiencias que construyen nuevos territorios posibles. La intervención visual fue muy bien recibida por parte de agentes del distrito, como por ejemplo el AMPA de la escuela donde se pintó, y se hizo difusión en las redes sociales y en la televisión local. A pesar de que las intervenciones visuales que se realizan en la calle pueden tener un carácter efímero, se trata de acciones que si se realizan en espacios urbanos abiertos pueden permanecer visibles durante un periodo prolongado de tiempo, infundir un sentido de pertenencia y de responsabilidad hacia estos espacios. Estas cuestiones representan una potencialidad educativa si se toma en consideración que la existencia de pautas de vinculación mutua y una geografía que incorpore significados de pertenencia son uno de los ejes de la EC (Essomba y Leiva, 2015).

Segundo, estos procesos de EC promueven el empoderamiento juvenil porque involucran las personas jóvenes en procesos de toma de decisiones sobre diseño, planificación e implementación de acciones en las cuales tienen un rol activo. Concretamente, esto se consigue a través del fomento del protagonismo de las personas jóvenes como agentes en los procesos comunitarios. Hay que dejar de considerar el graffiti y el arte urbano como un entretenimiento para poner en valor la posibilidad de establecer relaciones y vínculos con la comunidad. Se trata de procesos que se enmarcan en la reivindicación del derecho a la ciudad, que borran la frontera entre los usos privados y públicos de los espacios urbanos y que invitan a tomar un rol activo en la transformación. Sin embargo, contrariamente a lo que se había previsto inicialmente, la mayor transformación no se dio en los grupos de jóvenes, sino en los agentes técnicos participantes. Estos últimos asumieron la hipótesis general de la investigación y cambiaron la manera como promueven la EC a través del graffiti y el arte urbano en procesos educativos posteriores a la investigación.

En tercer lugar, las relaciones entre los agentes participantes mejoraron cuantitativa y cualitativamente durante la investigación. Los aspectos más destacados por los equipos técnicos fueron: el fortalecimiento de las relaciones con agentes de la comunidad que llevan a cabo una tarea cultural en el ámbito de la pintura callejera; la suma de líneas de acción educativa entre grupos de jóvenes; y el establecimiento de relaciones con jóvenes de la comunidad interesados en el graffiti y el arte urbano que previamente no frecuentaban los servicios y entidades participantes. Así, aumentó la coherencia y eficiencia de las acciones educativas.

Por otro lado, la investigación pretendía no reproducir sesgos de género, especialmente en relación con la participación de personas que no se identifican con las masculinidades hegemónicas. A pesar de que se intentó que las jóvenes participaran del mismo modo que los jóvenes, no se consiguió. En parte, esto se debe a que el graffiti y el arte urbano no dejan de darse en espacios urbanos que están subordinados al poder simbólico masculino. Las jóvenes participaron menos y les costaba encontrar su 
lugar cuando se realizaban acciones educativas más cercanas al graffiti tradicional. Por lo tanto, no se trata solo de fomentar la participación de mujeres en las acciones educativas, sino que hay que cambiar la manera como se promueven las acciones. Como se ha apuntado anteriormente, la adopción de acciones específicas, como por ejemplo la incorporación de referentes femeninos y la diversificación de lenguajes visuales y plásticos; comportó una mejora a la hora de contrarrestar estos sesgos.

Por último, también se identificaron nuevas líneas de investigación que están relacionadas con algunas limitaciones del proceso expuesto. Una de las líneas tiene que ver con la definición de estrategias para conseguir que el liderazgo colaborativo de los procesos de EC no sea asumido únicamente por los agentes técnicos de las comunidades, sino también por los agentes ciudadanos que las conforman. Segundo, hay que explorar formas y propuestas para prolongar los procesos de I-A más allá de un ciclo de acción y reflexión, especialmente en relación con los procesos de construcción de conocimiento. Finalmente, hay que definir estrategias para evitar que los procesos educativos de graffiti y arte urbano se conviertan en una manera de cooptar determinadas acciones consideradas como «problemáticas» y transformarlas en actividades «normalizadas». En síntesis, las contribuciones presentadas en este artículo aportan evidencias de las potencialidades educativas que conlleva que las personas jóvenes ejerzan sus derechos de transformar creativamente las ciudades donde viven.

\section{Referencias bibliográficas}

Abarca, J. (2010) El postgraffiti, su escenario y sus raíces: Graffiti, punk, skate y contrapublicidad (Tesis doctoral no publicada). Madrid, Universidad Complutense de Madrid.

Abarca, J. (2012). Introducción a los temas tratados. Recuperado (15/04/2019) de: http:// www.urbanario.es/

Aramburu, M. (2009). Convivència intercultural a l'espai públic urbà. Barcelona Societat: revista d'informació $i$ estudis socials, 16, pp. 62-70.

Augé, M. (1992). Los «No Lugares» Espacios del Anonimato: Una Antropología de la Sobremodernidad. Barcelona, Gedisa.

Austin, J. (2001). Taking the train: How graffiti art became an urban crisis in New York City. Nueva York: Columbia University Press.

Berti, G. I. (2010). Práctica cultural comunitaria. El Hip Hop. (Tesi doctoral no publicada). Universitat Autònoma de Barcelona, Barcelona.

Chang, J. (2007). Can’t Stop Won't Stop: A History of the Hip-Hop Generation. Reading, Ebury Press.

Civís, M. (2005). Els projectes educatius de ciutat com a praxi de desenvolupament comunitari de gènesi socioeducativa: Anàlisi i interpretació de la dimensió socioeducativa i sociocomunitària de tres projectes educatius de ciutat de Catalunya (Tesis doctoral no publicada). Universitat Ramon Llull, Barcelona.

Civís, M. y Riera, J. (2007). Nueva pedagogía comunitaria: Un marco renovado para la acción. Barcelona, Nau Llibres.

Civís y Longás, J. (2015). La colaboración interinstitucional como respuesta al desafío de la inclusión socioeducativa: Análisis de 4 experiencias de trabajo en red a nivel local en Cataluña. Educación XX1, 18, 1, pp. 213-236. 
Clack, A. (2010). Young Children as Protagonists and the Role of Participatory, Visual Methods in Engaging Multiple Perspectives. American Journal of Community Psychology, 46 (1-2), pp. 115-123.

Comas, D. (2000). Agobio y normalidad: una mirada crítica sobre el sector «ocio juvenil» en la España actual. Revista de estudios de Juventud, 50, pp. 9-22.

Comas, D. (2011). ¿Por qué son necesarias las políticas de juventud? Revista de estudios de juventud, 94, pp. 11-28

Comas, D. (2015). La emancipación de personas jóvenes en España: el túnel del miedo. Revista del Centro Reina Sofia sobre Adolescencia y Juventud, 2, pp. 7-24.

Dickinson, M. (2008). The making of space, race and place: New York City's war on graffiti,1970-the present. Critique of Anthropology, 28(1), pp. 27-45.

Duarte, C. (2012). Sociedades adultocéntricas: sobre sus orígenes y reproducción. Revista Última Década, 36, pp. 99-125.

Essomba, M. A. y i leiva, L. (2015). Educación comunitaria intercultural. Barcelona, Obra Social "La Caixa".

Feixa, C. (1998). De jóvenes, bandas y tribus: antropología de la juventud. Barcelona, Ariel.

Figueroa, F. (2014). El grafiti de firma: un recorrido histórico-social por el grafiti de ayer y de hoy. Madrid, Minobitia.

Freire, P. (1970). Pedagogía del oprimido. Madrid, Siglo XXI.

Grané, P. (2018). El dominio masculino en la pintura callejera. Recuperado (15/04/2019) de: http://www.ensayosurbanos.com/2018/10/06/el-dominio-masculino-en-la-pintura-callejera/

Grané, P.Rifà-Valls, M. y Essomba, M. À. (2017). Educación comunitaria a través de las artes: hacia una etnografía visual del graffiti y del arte urbano con jóvenes. Arteterapia: Papeles de arteterapia y educación artística para la inclusión social, 12, pp. 61-78.

Juanola, R. y Fàbregas, A. (2011). Mapping Roses: Un proyecto de aprendizaje Servicio en patrimonio cultural. Educación Artística Revista de Investigación, 2, pp. 123-128.

Keith, M. (2005). After the Cosmopolitan?: Multicultural Cities and the Future of Racism. Oxon y Nueva York, Routledge.

Lewin, K. (1946). Action research and minority problems. Journal of social issues, 2, pp. 34-46.

Latorre, G. (2008) Walls of empowerment: Chicana/o Indigenist Murals of California. Austin, University of Texas Press.

Lombard, KJ. (2014). Wildstyle women female hip hop graffiti. Artlink, 34(1), pp. 22-24

López, M. (2015) Indicadores sobre prácticas artísticas comunitarias: algunas reflexiones. En Arteterapia: Papeles de arteterapia y educación artística para la inclusión social, 10, pp. 209-234.

Marí, R. (2014). Ciudad y civilidad: el espacio público y sus configuraciones y regulaciones pedagógicas. Dentro de Ll. Ballester, B. Pascual i C. Vecina, Comunidad, trabajo en red e intervención socioeducativa, pp. 17-42. Palma, Edicions UIB.

Macdonald, N. (2001). The grafffiti subculture: Youth, masculinity and identity in London and New York. Houndmills, Palgrave MacMillan.

Marcellán-Barace, I., Lander, C., Aguirre, I. Y Arriaga, A. (2013). Estudio sobre jóvenes productores de cultura visual: evidencias de la brecha entre la escuela y la juventud. Arte, Individuo y Sociedad, 25, 3, pp. 524-535.

Mayugo, C., Pérez, X. y Ricart, M. (2004). Joves, Creació i Comunitat. Barcelona, Fundació Jaume Bofill.

Mcauliffe, C. (2012). Graffiti or Street Art? Negotiating the moral geographies of the creative city. Journal of Urban Affairs, 34, 2, pp. 189-206. 
Mcniff, J. (1988). Action research: principles and practice. Houndmills, MacMillan Education. Menor, L. (2015). Graffiti, Street Art, and Culture in the era of the Global City: The Ana Botella Crew case. Street Art \& Urban Creativity, 1(2), pp. 59-69.

Moreno, S. (2015). Anàlisi de les transicions juvenils des de la perspectiva de gènere: entre el pes del cicle de vida i el canvi generacional. Recerca, revista de pensament $i$ anàlisi, 16, pp. 111-138.

Palacios, A. (2009). El arte comunitario: origen evolución de las prácticas artísticas colaborativas. Arteterapia: Papeles de arteterapia y educación artística para la inclusión social, 4, pp. 197-211.

Pink, S. (2006). The Future of Visual Anthropology: Engaging the senses. Nueva York, Routledge.

Recinos, E. (2015). Is Street Art Sexist? Recuperado (15/04/2019) de: http://www.artslant. com/9/articles/show/41661

Rodó-De-Zárate, M. (2013). Gènere, cos i sexualitat: La joventut, l'experiència i l'ús de l'espai públic urbà. En Papers, 98, 1, pp. 127-142.

Sánchez De Serdio, A. (2008). Prácticas artísticas colaborativas: el artista y sus socios invisibles. DHuarte, 3, pp. 17-18.

Sany (2016). Girl Power [DVD]. Recuperado (15/04/2019) de: http://www.girlpowermovie. com/

Serracant, P. (2013) (Coord.). Enquesta a la joventut de Catalunya 2012. Volum 1: Transicions juvenils i condicions materials d'existència. Barcelona, Departament de Benestar Social i Família. Generalitat de Catalunya.

Tort, A. y Collet, J. (2017). Escola, famílies i comunitat. Barcelona, Octaedro.

Úcar, X. (coord.) (2009). Enfoques y experiencias Internacionales de acción comunitaria: en España, Israel, Finlandia, Estados Unidos de América y Brasil. Barcelona, Graó.

Úcar, X. Jiménez-Morales, M., Soler, P. I Trilla, J. (2016). Exploring the conceptualization and research of empowerment in the field of youth. International Journal of Adolescence and Youth. Recuperado (15/04/2019) de: http://dx.doi.org/10.1080/02673843.2016.1209 120

Wacławek, A. (2011). Graffiti and Street Art. Londres, Thames \& Hudson Ltd.

Zegrí, M. (2014). Mediación y conflictos urbanos: Experiencias de mediación en espacios públicos con jóvenes, comunidades de vecinos y comercios. Barcelona, Icaria Milenrama. 